\title{
Investigation and Analysis of the Canine Oral Health Care Situation
}

\author{
$\mathrm{Na} \mathrm{Li} i^{1, a, *}$ \\ ${ }^{1}$ Binzhou Polytechnic, Binzhou 256600, China \\ a951011580@qq.com
}

\begin{abstract}
Animal's teeth may have certain kinds of lesion during their growth and development, the result of which is a variety of dental diseases. Based on this situation, the writer has managed to investigate on the district center for disease control, which included 126 oral health care cases in animals. Through the analysis of the clinical data and relative canine oral health and canine teeth disease problems, this article has put forward solutions to prevent animals from suffering from common oral diseases and dental diseases.
\end{abstract}

Keywords: Canine branch; Oral health care; Tooth disease; Prevention strategies

\section{Introduction}

It has been shown in clinical trials that common dental diseases include gingivitis, tooth stones and tooth follicles. There is a fundamental link between the causes of canine dental disease and the relationship between food granules, epithelial cells, and oral saliva. There are many kinds of canine dental diseases, and the most common one is dental plaque dental disease. This type of dental disease is characterized by the rapid rate of disease and the wide area of infection. In general, dental plaque can occur after a few hours of dental cleaning. Plaque is not easy to remove, even in the way of drinking water. Dental plaque has been in the oral cavity for a long time, and the result is the formation of dental plaque.

\section{Common Sense of Dental Disease}

Normally, the 2nd to the 8th month is a critical period for the formation of animal teeth. According to the survey data, the animals usually has 28 baby teeth, and when the baby tooth grows to a certain period of time, the permanent teeth will appear, and the number of permanent teeth will be 42. Large animals are much more likely to have dental problems than small animals. The health of the canine teeth is partly influenced by their personality. For example, the use of the tooth and the activity frequency of the animal with lively personality is relatively high, and the wear degree of the tooth is deeper, sometimes even cause fracture.

\section{Analysis of Common Basic Disease in Canine Oral Cavity}

After the analysis of 126 canine oral health related data collected from the animal disease control center of the region, we can conclude that the key factor for the formation of canine teeth is the long-term use of teeth to obtain food. In the process of getting food, any animal can't help without the chewing of the tooth, especially the canine. The process of chewing food increases the frequency of the tooth's use, causing bacteria to be present around the teeth, and the bacteria can reproduce further. The presence of dental plaque in the mouth of a animal can secrete a substance 
that causes gum inflammation called enzymes, and the presence of enzymes is the main cause of periodontitis. Many canines breeder lack of professional knowledge, so they can't choose the suitable feed type for the growth of the animals. A lot of feed lack of phosphorus, calcium and other substances, which is also the important cause for the canine diseases. In addition, many breeders will train their animals, and the training methods will have a variety of characteristics, but they will inevitably include the exercise of pulling items. In the process of pulling, excessive force will increase the pressure of the canine teeth, causing the canine teeth to loosen and cause a series of dental diseases.

\section{Clinical Symptoms of Canine Teeth Disease}

The analysis of canine tooth disease can be done by the following two methods. The first method is to observe the abnormal characteristics of canine teeth from the surface of the teeth, such as bad breath, a salivation situation and dental plaque on canine teeth. The second method is to observe the eating situation of the animal. If the feeding of the animal is mainly liquid or soft food, it indicates that the animal may have some health problems. If the animal is not able to chew the food during the meal, it shows that there is a problem with the canine teeth, and this is the time to consider whether there is some form of dental disease. If the animal has no appetite and is wasting away, the disease may be worsening.

\section{Common Oral Dental Disease and Treatment}

Double Row of Teeth. Also known as the baby teeth not taken off, double row teeth is most common in small animals (such as: Yorkshire, poodles, etc.). It is commonly caused by the retention of baby teeth. The existence of deciduous tooth can affect the growth of the permanent teeth, and food scraps and hair easily accumulate in deciduous tooth position. If the deciduous teeth are not removed, it can easily lead to a large accumulation of calculus, which can lead to gingivitis and periodontitis. The common symptoms of deciduous tooth are bad breath, disorderly bite, dare not to eat, and so on. Therefore, in order to avoid causing trouble to canine oral health, animals should be timely taken to normal pet hospital to pull up baby teeth through professional skilled doctors using regular milk special forceps .

Dental Calculus. The chewing ability of canine teeth depends greatly on the degree of hardness of the type of feeding. It is also the main cause of tooth calculi. If the animal has a long-term diet of soft meat, the friction between the food and the canine teeth will decrease, which will lead to a decrease in the chewing ability of the canine teeth. If food residues persist in the mouth and around the gums, it can form a plaque in time.

The key to solving the problem of canine dental calculus is to grasp the causes of dental calculus and know the specific shape of the dental calculus. If the color is dark brown or light yellow, the animal's mouth is prone to bad breath, and it can lead to gum bleeding and even periodontal disease. At this time, the animal's appetite will be affected, and the desire of eating will gradually decrease. Once this happens, professional treatment is necessary. The tooth calculi is not impossible to remove. Doctors can use the ultrasound to clean the tooth, but they should grasp the yardstick in the cleaning process so as to avoid causing secondary injury. Cleaning can begin with one side, and then move to the other side. If doctors find teeth have been loose in the process, they can take out the teeth. Be sure to disinfect the mouth after cleaning work. In addition, because the cleaning of the teeth is very complicated, it will bring some pain to the animals during the cleaning 
process, so the animal can be treated with anesthesia before cleaning. In determining the animal's dental calculus has been completely removed, the anesthetic can be removed. Then, with a certain amount of pharmacological action, the animal's teeth can be restored to a healthy state and the animal's ability to chew can be improved.

Gingivitis. If the animal's dental calculus is not well solved and the animal's oral cavity is not clean, it can lead to an infection in the dental cavity of the animal. Oral cavity tooth infection has a great pathological and survival risk. Without timely treatment, it can pose a great threat to the kidney function of the animal, resulting in the occurrence of various kidney diseases. Relevant clinical data showed that animals with gum disease have problems such as loss of appetite, swollen gums, bad breath. Because every animal's health level and body quality is different, some can produce chronic gingivitis phenomenon, the consequence of which is very serious. The specific situation needs to be analyzed in detail according to the different characteristics of each animal.

The causes of gingivitis is that the animal's teeth problems aren't carefully solved, animal oral lining residue has not been cleaned, which in turn cause infection to teeth. So when solving the problem of gingivitis, we should have full analysis of the main reasons in order to find a solution to the problem. It is necessary to have local anesthesia in cleaning. After anesthesia, we should analyze gum infection status, and check out the problem that cause gum infection. If gum infection is fresh wounds, the first step is to disinfect animal mouth, and then deal with necrotic tissue; If the root of the canine teeth has been damaged, the damaged teeth should be removed and replaced with new teeth. For those animals who have serious dental disease, it is necessary to seek medical treatment in time and to conduct management in professional institutions. The host also need to pay extra attention when they're feeding. They should try to get some fluffy food to make sure the wound is healed better.

Tooth Fold. In the case of gravity, the canine teeth can break. The main causes of tooth folding include feeding and biting. Crown fracture is the main form of canine tooth folding, and the examination of tooth folding requires the use of $\mathrm{X}$ ray to grasp the fracture position. The animal has a great pain in the process of examination and treatment, so the animal needs to be anesthetized before being examined. We have no way to remedy completely broken teeth, so we can only pull it out, and then to suture the affected area. If teeth are not completely broken, we do not need to pull teeth out. They may be kept by the remedy of the gum tissue. Because the teeth is not very strong, we can use the stainless steel braces to fix the teeth. Choose foods rich in calcium and phosphorus when feeding and monitor their canine health in real time. Animals should be based on food and soft food in the stage of canine tooth recovery.

Periodontal Disease. Inflammation caused by periodontal tissue in the oral cavity is usually accompanied by gingivitis. The symptoms of canine expression are bad breath, gingiva red swelling, severe tooth loss of gingiva, dissolution of alveolar bone, abscess in tooth cavity, loose tooth, etc. The animal's mouth is usually sensitive. There will be a loss of appetite, eating soft food, not wanting to chew toys. With the deepening of canine teeth infection, teeth falling off, facial fistula and even mandibular fracture will appear. It can also lead to myocarditis and the incidence of renal disease. In order to avoid the aggravation of illness, the symptoms should be treated in time.

\section{Prevention and Health Care Methods for Canine Dental Diseases}

The incidence of tooth disease is very common and the cycle is short, so animals should be taken regularly to the professional hospital for diagnosis and treatment. This can effectively remove the animal's tartar and the lining of the oral cavity, which greatly reduces the incidence of tooth 
disease in animals. In order to ensure the ability of the animal to chew, we should not only feed them soft food, but also exercise the bite force of the animal in the ordinary games, which will help to clean the dirt in the animal's mouth. Don't use bones to train a animal's bite because the bone is so hard that it will damage the canine teeth.

Brushing teeth regularly is also an effective way to ensure healthy canine teeth. However, it is worth noting that brushing cannot be rushed. On the contrary, it should be gradual. In the beginning we can put the finger on the surface layer of fangs and then gently move the fingers back and forth. It is suggested that we take good control of time. It should not be too long. The aim is to let the animals adapt to this operation, and gradually we can finish this brushing operation. The order of brushing is also very important, which is to press from outside to inside. In the choice of toothpaste, we had better not choose the toothpaste that is suitable for animals.

\section{Reference:}

[1] C.H. Li and W. He. The Prevention and Treatment of Dental Diseases of Pet Animals [J]. Animal husbandry veterinarian, 2017, (3) : 39-40.

[2] J.Qi and Z.F.Xia. Oral Health and Nutrition of Animals [C].

[3] Common Teeth and Oral Diseases of Animals [J]. China Working Animal Industry, 2010,(1):44. 